\title{
BMNeurology open May lamotrigine be an alternative to topiramate in the prevention of migraine with aura? Results of a retrospective study
}

\author{
Carmelo Luca Smeralda, ${ }^{1,2}$ Gian Luigi Gigli, ${ }^{1,3}$ Francesco Janes, ${ }^{1}$ \\ Mariarosaria Valente $e^{1,2}$
}

To cite: Smeralda CL, Gigli $\mathrm{GL}$, Janes $\mathrm{F}$, et al. May lamotrigine be an alternative to topiramate in the prevention of migraine with aura? Results of a retrospective study. BMJ Neurology Open 2020;2:e000059. doi:10.1136/ bmjno-2020-000059

Received 23 March 2020 Revised 01 July 2020 Accepted 08 July 2020
Check for updates

(C) Author(s) (or their employer(s)) 2020. Re-use permitted under CC BY-NC. No commercial re-use. See rights and permissions. Published by BMJ.

${ }^{1}$ Clinical Neurology, Udine University Hospital, Udine, Italy ${ }^{2}$ DAME, University of Udine, Udine, Italy

${ }^{3}$ DMIF, University of Udine, Udine, Italy

Correspondence to Dr Carmelo Luca Smeralda; carmelosmeralda@gmail.com

\section{ABSTRACT}

Background Evidence suggests that lamotrigine could be effective in reducing aura frequency and duration. However, studies comparing lamotrigine to other, first-line prophylactic agents solely involving patients suffering from migraine with aura are still lacking. The aim of this study was to compare the efficacy of lamotrigine and topiramate for the preventive treatment of migraine with aura. Methods Fifty-three patients suffering from migraine with aura treated with lamotrigine or topiramate for at least 6 months were included. Pre- and post-treatment clinical data regarding monthly aura frequency and duration, monthly migraine frequency, days of headache and rescue medication used per month were collected.

Results Responder rates were similar between the two treatment groups at 6-month follow-up. Interestingly, responder rates for aura frequency and duration were higher in the lamotrigine group compared with the topiramate group ( $88 \%$ vs $79 \%$ and $73 \%$ vs $54 \%$ ). Moreover, $50 \%$ of the lamotrigine-treated patients reported a complete disappearance of migraine aura compared with $37 \%$ of topiramate-treated patients. Side effects were more frequent in topiramate group compared with lamotrigine group $(p=0.004)$.

Conclusions Lamotrigine should be considered in clinical practice for the preventive treatment of migraine with aura especially for patients reporting prolonged aura and who do not respond, have contraindications or discontinue topiramate treatment due to side effects.

\section{INTRODUCTION}

Migraine is a common disabling neurological disorder, characterised by unilateral, pulsating, severe headache attacks, frequently associated with autonomic symptoms such as nausea, vomiting, photophobia and phonophobia. In approximately $30 \%$ of cases, migraine is preceded by or associated with transient, reversible, focal neurological symptoms called aura, ${ }^{1}$ which develops gradually over 5-20 min and usually lasts for less than an hour. ${ }^{2}$ The most common aura symptoms are visual and sensory ones, followed by language, motor and brainstem disturbances. ${ }^{3}$
Pathophysiology of migraine aura has been linked to cortical spreading depression (CSD), which consists in a self-propagating wave of depolarization, followed by a more slowly-propagating wave of neuronal inhibition usually developing within the occipital cortex and then spreading throughout more anterior cortical areas at a speed of $3-6 \mathrm{~mm} /$ min. ${ }^{4}$ According to experimental models, CSD is characterised by changes in extracellular potassium concentrations and neuronal glutamate release, which determine the propagation of the depolarization wave. ${ }^{5}$ Perfusion weighted and functional MRI studies showed a causal relationship between CSD and aura within human visual cortex highlighting CSD causative role in determining the aura phenomenon. ${ }^{6-8}$

Latest evidence suggests that CSD can also determine meningeal nociceptor firing, thus leading to the activation of the trigeminovascular system which has been linked to the development of migraine headache. ${ }^{9}$ However, the relationship between CSD and migraine headache is not fully understood yet.

Recent developments in the understanding of migraine pathophysiology have led researchers to investigate new targeted therapeutic approaches for the treatment of the disease. Data from randomised, controlled trials exploring the efficacy of monoclonal antibodies for the treatment of migraine showed promising results and would probably change the management and the clinical outcome of patients affected by episodic and chronic migraine in the next years. ${ }^{10}$ Nevertheless, clinical management of migraine with aura remains extremely uncertain for neurologists since preventive treatments showing a clear efficacy in reducing aura frequency and duration are still lacking. In fact, currently 
approved first-line agents for migraine prophylaxis, showed little or no benefit for the preventive treatment of migraine with aura. ${ }^{11}$

Lamotrigine is an antiepileptic, sodium channel blocking drug able to induce an indirect inhibition of neuronal glutamate release thus blocking CSD propagation throughout the cerebral cortex as shown by experimental model of CSD. ${ }^{12}$ The role of lamotrigine for the preventive treatment of migraine has been previously investigated. A double-blind, randomised,controlled, crossover study involving suffering from migraine with and without aura, concluded that lamotrigine was ineffective in reducing migraine frequency and intensity compared with topiramate, another antiepileptic drug approved as first-line agent for migraine prophylaxis, ${ }^{13}$ although efficacy outcomes of patients solely affected by migraine with aura were not reported at the end of the abovementioned study. On the other hand, evidence suggests that lamotrigine could be effective within the migraine aura clinical setting. ${ }^{14-16}$ To our best knowledge, studies comparing lamotrigine to others, first-line prophylactic migraine agents involving a population of patients solely suffering from migraine with aura are still lacking. For this reason, we conducted a retrospective analysis in order to assess the efficacy of lamotrigine compared with topiramate for the preventive treatment of migraine with aura.

\section{MATERIAL AND METHODS}

We reviewed the clinical records of 1152 patients referred to our Headache Clinic from January 2014 to February 2019. Inclusion criteria for the enrolment were: a diagnosis of migraine with aura or aura without migraine according to the last Headache International Society diagnostic criteria; ${ }^{2}$ aged $\geq 18$ years; migraine onset before the 50 years of age; presence of migraine attacks for at least 1 year prior to entering the study; at least two attacks of migraine with aura or aura without migraine per month in the previous 3 months. Exclusion criteria were: any other non-migraine headache; chronic headache defined as more than 15 headache days per month; coexistence of medication overuse headache; any other severe co-morbid medical condition (e.g. cardiac, hepatic, renal and psychiatric disease); other preventive migraine treatments in the last 3 months prior to entering the study; previous failure of more than two prophylactic agents. Patients allocation to topiramate or lamotrigine treatment was based on neurologist's clinical judgement, taking into account patient clinical scenario, possible contraindications to the drugs, previous ineffective preventive treatments as well as patient preferences.

A total of 53 patients were recruited, 25 of whom treated with topiramate and 28 with lamotrigine. Nine patients within the topiramate group and six patients within the lamotrigine group also reported migraine without aura attacks. Finally, one patient affected by aura without migraine was also included, who had been treated with topiramate. Local ethics committee waived the need for approval and the need to obtain consent for the collection, analysis and publication of the retrospectively obtained and anonymized data for this study.

According to clinical records, all patients had normal extensive laboratory findings, including workup for an underlying coagulopathy. Furthermore, all of them have previously undergone MRI brain scan in order to exclude secondary cause of headache. Treatment dosage varied between $25 \mathrm{mg}$ two times a day and $100 \mathrm{mg}$ two times a day both for topiramate and lamotrigine, according to clinical judgement. Treatments were gradually started in order to reduce development of side effects. All patients were followed for a period of at least 6 months after starting treatment. Reassessment took place on ambulatory visit or on phone interviews conducted by trained examiners. Data collection was based both on patient self-reporting and on examination of headache diary. We evaluated responder rates, defined as the percentage of patients reporting a reduction of at least $50 \%$ of migraine with aura attacks at the end of the follow-up period, and optimal responder rates, defined as patient reporting a complete disappearance of migraine with aura attacks at 6 month follow-up. We further evaluate the pre- to posttreatment change of monthly (28 days) aura frequency, aura duration per episode (minutes), monthly (28 days) migraine frequency, monthly days of headache and rescue medication used per month between the two treatment groups. Finally, dropout rate and type and frequency of side effects for both treatment groups were reported.

\section{STATISTICAL ANALYSIS}

Statistical analysis was performed using SPSS V.20 (SPSS, Chicago, Illinois, USA). A Shapiro-Wilk's test (W statistic $=0.74 ; \mathrm{p}=0.001)$ and a visual inspection of histograms, normal $\mathrm{Q}-\mathrm{Q}$ plots and box plots showed that samples pretreatment data for all the variables taken into account were not normally distributed for both treatment groups. Pretreatment descriptive statistics for both groups and pre- to post-test analysis were then performed using non-parametric tests. We used the Mann Whitney U test for continuous variables and the $\chi^{2}$ test for nominal and ordinal variables. A two-tailed $\mathrm{p}$ value was calculated for each test. A significance level of $<0.05$ was set.

\section{RESULTS}

Of the 53 patients recruited, 25 (3 male/22 female) were treated with topiramate and 28 ( 3 male/25 female) with lamotrigine. Among the two treatment groups, only one patient affected by aura without migraine was included, who had been treated with topiramate. Demographics and baseline clinical characteristics of both treatment groups are detailed in table 1.

Clinical features and distribution of migraine aura within the study population are reported in table 2 . 
Table 1 Demographics and baseline clinical characteristics of study population

\begin{tabular}{|c|c|c|c|c|c|}
\hline & \multicolumn{2}{|c|}{ Topiramate } & \multicolumn{2}{|c|}{ Lamotrigine } & \multirow{2}{*}{$\begin{array}{l}\text { P value }(<0.05) \\
0.60\end{array}$} \\
\hline $\operatorname{Sex}(M / F)(\%)$ & $3 / 22$ & $(12 / 88)$ & $3 / 25$ & $(11 / 89)$ & \\
\hline Median age (years, range) & 46 & $(23-66)$ & 37 & $(18-84)$ & 0.021 \\
\hline $\begin{array}{l}\text { Median age of migraine onset (years, } \\
\text { range) }\end{array}$ & 18 & $(6-48)$ & 15.5 & $(6-38)$ & 0.058 \\
\hline \multirow[t]{2}{*}{ Prior preventive treatment (\%yes/\%no) } & $12 / 25$ & $(48 / 52)$ & $20 / 28$ & $(71 / 29)$ & 0.082 \\
\hline & Median & Range & Median & Range & \\
\hline Aura episodes per month & 4 & $1-15$ & 4.5 & $1.5-15$ & 0.18 \\
\hline Duration of aura episodes (min) & 28 & $5-90$ & 30 & $5-90$ & 0.44 \\
\hline Migraine attacks per month & 7 & $2.5-15$ & 5 & $1.5-15$ & 0.15 \\
\hline Days of migraine per month & 9 & $1.8-15$ & 7.5 & $1.8-15$ & 0.35 \\
\hline Rescue medication used per month & 10 & $2.5-15$ & 9 & $1.5-15$ & 0.51 \\
\hline
\end{tabular}

Groups were sex-but notage-matched, since lamotriginetreated patients were younger than those treated with topiramate (median age 37 and 46 years, respectively). There was no difference in age of migraine onset between the two groups. Interestingly, among lamotrigine-treated patients, $71 \%(20 / 28)$ had been previously treated with at least another migraine prophylactic agent compared with $48 \%(12 / 25)$ of those within the topiramate group, although this difference was not statistical significant, as shown in table 1 .

Median prescribed dosage was $50 \mathrm{mg}$ two times a day for both lamotrigine and topiramate (range $25 \mathrm{mg}$ two times a day to $100 \mathrm{mg}$ two times a day). Two patients treated with lamotrigine and one patient treated with topiramate discontinued treatment after few days due to severe side effects and were therefore excluded from outcome analysis.

Responder rates and optimal responder rates for monthly aura frequency, aura duration per episode and monthly migraine frequency are detailed in table 3 .

Both lamotrigine and topiramate were highly effective in reducing monthly migraine frequency of at least $50 \%$ at the end of the follow-up period and no substantial difference between the two treatment groups was reported. Responder rates for aura frequency and

Table 2 Clinical characteristics and distribution of migraine aura within the two treatment groups

\begin{tabular}{lcc}
\hline & Frequency & Per cent \\
\hline Topiramate & 23 & 92 \\
\hline Visual & 2 & 8 \\
\hline Visual and sensory & 20 & 71 \\
Lamotrigine & 1 & 3 \\
\hline Visual & 4 & 14 \\
\hline Hemiplegic & 3 & 10 \\
\hline Visual and sensory & & \\
\hline $\begin{array}{l}\text { Visual, sensory and } \\
\text { aphasic }\end{array}$ & & \\
\hline
\end{tabular}

duration were higher in the lamotrigine group compared with the topiramate group (88\% vs $79 \%$ and $73 \%$ vs $54 \%$, respectively), although these differences failed to reach statistical significance.

Similarly, optimal responder rates, defined as patient reporting a complete disappearance of migraine attacks and migraine aura, tended to be higher in lamotrigine compare to topiramate-treated patients $(27 \%$ vs $13 \%$ and $50 \%$ vs $37 \%$, respectively).

We further evaluated pre- to post-treatment changes between the two groups for all the parameters taken into account (table 4).

While change in monthly migraine frequency, number of migraine days and rescue medication used per month were comparable between the two treatment groups, a slightly more pronounced reduction of monthly aura frequency was found within the lamotrigine group compared with the topiramate group.

Interestingly, lamotrigine showed to reduce aura duration per episode of $15 \mathrm{~min}$ while topiramate show no relevant efficacy in reducing this parameter $(\mathrm{p}=0.062)$.

As mentioned above, two patients within the lamotrigine group and one patient within the topiramate group discontinued treatment after few days due to severe

Table 3 RRs and ORRs at the end of the follow-up period

\begin{tabular}{llll}
\hline & Topiramate & Lamotrigine & $\begin{array}{l}\text { P value } \\
(<0.05)\end{array}$ \\
\hline RRs & & & \\
\hline Aura frequency & $19 / 24(79 \%)$ & $23 / 26(88 \%)$ & 0.37 \\
$\begin{array}{l}\text { Aura duration } \\
\text { Migraine }\end{array}$ & $13 / 24(54 \%)$ & $22 / 26(73 \%)$ & 0.16 \\
$\begin{array}{l}\text { frequency } \\
\text { ORRs }\end{array}$ & $20 / 23(87 \%)$ & $17 / 26(85 \%)$ & 0.81 \\
\hline Aura & & & \\
\hline Migraine & $9 / 24(37 \%)$ & $13 / 26(50 \%)$ & 0.37 \\
\hline
\end{tabular}

ORRs, optimal responder rates; RRs, responder rates. 
Table 4 Pre- to post-treatment changes between the two groups at follow-up

\begin{tabular}{|c|c|c|c|c|c|}
\hline & \multicolumn{2}{|c|}{ Topiramate } & \multicolumn{2}{|c|}{ Lamotrigine } & \multirow{2}{*}{$\begin{array}{l}\text { P value } \\
(<0.05)\end{array}$} \\
\hline & Mdn & IQR & Mdn & IQR & \\
\hline Aura frequency per month & -2.4 & 3.4 & -4 & 3.9 & 0.28 \\
\hline Migraine attacks per month & -4.5 & 8 & -3.7 & 6.6 & 0.10 \\
\hline Days of migraine per month & -6 & 3 & -4.7 & 5.3 & 0.37 \\
\hline
\end{tabular}

IQR, interquartile range; Mdn, median.

side effects (rash for lamotrigine and cognitive symptoms for topiramate). Patients who continued both treatments reported only mild side effects. Within the topiramate group, the most frequent side effects reported were transient paresthesia, weight loss, dizziness, somnolence and cognitive symptoms such as concentration/attention difficulties, slow-thinking and mood changes. Among the lamotrigine-treated patients, the most reported side effects were fatigue, anxiety, somnolence and nausea (see table 5 for details). Side effects were significantly more frequent within the topiramate group compared with the lamotrigine group $(\mathrm{p}=0.004)$.

\section{DISCUSSION}

The aim of our study was to explore the role of lamotrigine for the prevention of episodic migraine with aura. For this purpose, we decided to compare lamotrigine to topiramate, an antiepileptic drug established to be highly effective for the preventive treatment of episodic migraine. ${ }^{17}$ Pooled data from three large multicentre, double-blind, placebo-controlled trials demonstrated a clinically relevant response of monthly migraine frequency in patients treated with topiramate compared with placebo and led to topiramate approval as firstline drug for migraine prophylaxis. ${ }^{18}$ Further studies confirmed a similar efficacy of topiramate compared with other first-line prophylactic drugs such as propranolol and valproate. ${ }^{19}$ Real-world experience with topiramate proved its efficacy in reducing migraine frequency, improving patient quality of life and social functioning as well as reducing healthcare resources allocation to headache,$^{20}$ making topiramate one of the most used migraine preventive drug in clinical practice nowadays. Moreover, due to its mechanism of action, topiramate is also believed to affect migraine aura initiation. ${ }^{21}$ In an experimental model of CSD, topiramate showed to inhibit the propagation of induced CSD in a dose-dependent manner. ${ }^{22}$ However, its clinical efficacy in preventing migraine aura is not clear. In an open longitudinal, pilot study involving patients affected by migraine with aura, topiramate failed to reduce aura frequency and duration at 6-month follow-up. Additionally, a posthoc analysis of the PROMPT trial, a well-designed study consisting of an open label phase in which patients were treated with topiramate for 26 weeks, followed by a double-blind phase in which patients were randomized to receive placebo or continue to receive topiramate, showed a significant reduction in migraine aura frequency at the end of the open label phase which was maintained throughout the double-blind phase. ${ }^{23}$ However, no significant change in number of auras between topiramate and placebo were found at the end of the follow-up period. ${ }^{24}$

Contrary to topiramate, the role of lamotrigine in the prevention of migraine is more controversial.

The North American guidelines for the preventive treatment of episodic migraine included lamotrigine among medications established to be ineffective. ${ }^{17}$ This low level of grading is essentially based on the results of

Table 5 Side effects and dropout rate between the two treatment groups

\begin{tabular}{|c|c|c|c|c|c|}
\hline & Topirar & & Lamo & & \\
\hline & $\mathbf{N}$ & $\%$ & $\mathbf{N}$ & $\%$ & $P$ value $(<0.05)$ \\
\hline Overall & $15 / 25$ & 60 & $6 / 28$ & 21 & 0.004 \\
\hline Weight loss & $6 / 25$ & 24 & & & \\
\hline Transient paresthesia & $9 / 25$ & 36 & & & \\
\hline Cognitive symptoms & $7 / 25$ & 28 & $1 / 28$ & 4 & \\
\hline Somnolence & $2 / 25$ & 8 & $1 / 28$ & 4 & \\
\hline Fatigue & & & $1 / 28$ & 4 & \\
\hline Rash & & & $2 / 28$ & 7 & \\
\hline Dropout due to side effects & $1 / 25$ & 4 & $2 / 28$ & 7 & 0.621 \\
\hline
\end{tabular}


two prospective, randomized, controlled trials, involving migraineurs both with and without aura. Both studies failed to demonstrate the efficacy of lamotrigine in migraine prophylaxis compare to placebo and topiramate respectively. ${ }^{13}$ However, posthoc analysis from the abovementioned studies, exploring the role of lamotrigine in reducing migraine aura frequency, was not conducted due to the small number of patients suffering from migraine with auraincluded. Lamotrigine is an antiepileptic drug that exerts its function by blocking voltage-sensitive sodium channels, indirectly leading to the inhibition of neuronal release of glutamate, a neurotransmitter involved in the development and propagation of CSD. Evidence suggests that chronic intraperitoneal administration of lamotrigine determine a strong suppression of CSD propagation in rats compared with placebo and valproate. ${ }^{12}$ More than a decade ago, several pilot, open label studies exploring the role of lamotrigine in the prophylaxis of migraine aura were conducted and pointed to a great efficacy of the drug in reducing both frequency and duration of aura episodes. ${ }^{14-16}$ Based on these findings, a prospective open study including 59 patients suffering from migraine with aura treated with lamotrigine showed a sustained reduction of aura frequency and duration. Additionally, more than three quarters of the aura responder patients also reported a significance reduction of migraine attacks frequency during the treatment period, suggesting a potential efficacy of lamotrigine for the prophylaxis of migraine headache as well. ${ }^{26}$ Based on these results, lamotrigine is largely used in clinical practice for the preventive treatment of migraine with aura especially in patients for whom other preventive treatments are ineffective or contraindicated, although clear evidence supporting the non-inferiority of lamotrigine compared with other approved migraine prophylactic agents is still lacking.

Our study is the first to compare the efficacy of lamotrigine and topiramate in solely patients suffering from migraine with aura. Both treatments showed to equally reduce monthly frequency of migraine attacks at 6 months of follow-up. Consequently, our results suggest a similar efficacy of both treatments within the clinical setting of migraine with aura. Interestingly, the majority of lamotrigine-treated patients included in our study had been previously treated with at least another migraine prophylactic agent, suggesting that the therapeutic effect of lamotrigine was certainly not obtained in a population of less resistant patients. Additionally, despite a well-established tolerability profile, discontinuation rates due to adverse events associated with topiramate are higher in patients with migraine compared with patients with epilepsy. ${ }^{27}$ Although the small number of patients included in our study, our tolerability data showed a significantly higher rate of adverse events within topiramate group compared with lamotrigine-treated patients.

Finally, according to the North American Antiepileptic Drug pregnancy registry, topiramate showed a dose independent threefold higher risk of congenital malformations and child developmental retardation in women exposed to the drug, especially in the first trimester of pregnancy. ${ }^{28}$ On the other hand, teratogenic activity of lamotrigine is dose-dependent and exerted at higher dosage than that used for migraine prophylaxis. ${ }^{29}$ Based on our findings, lamotrigine should be considered in clinical practice for patients having migraine with aura reporting unresponsiveness to topiramate treatment or who discontinue the drug due to side effects. Moreover, lamotrigine could be safely offered to patients who have contraindications to topiramate, especially migraine with aura women planning to have pregnancy.

Regarding the effects on aura frequency and duration, our results showed a trend towards a better efficacy of lamotrigine compared with topiramate. Responder rates for monthly aura frequency were similar between the two treatment groups. However, approximately half of the lamotrigine-treated patients reported a complete disappearance of aura at 6-month follow-up. Additionally, lamotrigine determined a reduction of aura duration per episode of approximately $15 \mathrm{~min}$. In line with previous findings,${ }^{16}$ lamotrigine should then be considered in clinical practice for the treatment of prolonged or disturbing migraine with aura. We are aware of several limitations of this study. A possible bias in patients' selection, the small number of patients recruited, the absence of a placebo controlled group and the retrospective nature of our study may have indicated a higher efficacy of both drugs than observed in other studies. Although results similar to ours for lamotrigine have been previously reported by Pascual et $a l^{16}$ with a retrospective, non-placebocontrolledì analysis, we think that the main result of our study was not the demonstration of the effectiveness of any specific drug, rather the evidence of a similar effectiveness of lamotrigine compared with topiramate. This could open new therapeutic possibilities, especially in patients for whom topiramate is contraindicated or who are intolerant to this drug. We hope that our observation may stimulate further prospective, double-blind, randomized, controlled trials to prove the role of lamotrigine in the prophylaxis of migraine with aura.

\section{CONCLUSION}

Lamotrigine is equally effective as topiramate for the preventive treatment of migraine with aura and exhibits a better tolerability profile. Lamotrigine should be considered in clinical practice especially for patients reporting prolonged aura and who do not respond, have contraindications or discontinue topiramate treatment due to side effects.

Contributors CLS and MV planned the study. CLS and FJ collected and interpreted the data and wrote the first manuscript draft. All authors participated in the writing of the final manuscript and all authors approved final manuscript. GLG was responsible for the overall supervision of the present work.

Funding The authors have not declared a specific grant for this research from any funding agency in the public, commercial or not-for-profit sectors. 
Competing interests None declared.

Patient consent for publication Not required.

Provenance and peer review Not commissioned; externally peer reviewed.

Data availability statement Deidentified participant data are available on reasonable request.

Open access This is an open access article distributed in accordance with the Creative Commons Attribution Non Commercial (CC BY-NC 4.0) license, which permits others to distribute, remix, adapt, build upon this work non-commercially, and license their derivative works on different terms, provided the original work is properly cited, appropriate credit is given, any changes made indicated, and the use is non-commercial. See: http://creativecommons.org/licenses/by-nc/4.0/.

\section{REFERENCES}

1 Foroozan R, Cutrer FM. Transient neurologic dysfunction in migraine. Neurol Clin 2019;37:673-94.

2 Headache Classification Committee of the International Headache Society (IHS). The International classification of headache disorders, 3rd edition (beta version). Cephalalgia 2013;33:629-808.

3 Kissoon NR, Cutrer FM. Aura and other neurologic dysfunction in or with migraine. Headache 2017;57:1179-94.

4 Somjen GG. Aristides Leão's discovery of cortical spreading depression. J Neurophysiol 2005;94:2-4.

5 Sugaya E, Takato M, Noda Y. Neuronal and glial activity during spreading depression in cerebral cortex of cat. $J$ Neurophysiol 1975;38:822-41.

6 Cutrer FM, Sorensen AG, Weisskoff RM, et al. Perfusion-Weighted imaging defects during spontaneous migrainous aura. Ann Neurol 1998;43:25-31.

7 Hadjikhani N, Sanchez Del Rio M, Wu O, et al. Mechanisms of migraine aura revealed by functional $\mathrm{MRI}$ in human visual cortex. Proc Natl Acad Sci U S A 2001;98:4687-92.

8 Cao Y, Welch KM, Aurora S, et al. Functional MRI-BOLD of visually triggered headache in patients with migraine. Arch Neurol 1999;56:548-54.

9 Levy D. Endogenous mechanisms underlying the activation and sensitization of meningeal nociceptors: the role of immuno-vascular interactions and cortical spreading depression. Curr Pain Headache Rep 2012;16:270-7.

10 Edvinsson L. Role of CGRP in migraine. Handb Exp Pharmacol 2019;255:121-30.

11 D'Andrea G, Nordera GP, Allais G. Treatment of aura: solving the puzzle. Neurol Sci 2006;27 Suppl 2:s96-9.

12 Bogdanov VB, Multon S, Chauvel V, et al. Migraine preventive drugs differentially affect cortical spreading depression in rat. Neurobiol Dis 2011;41:430-5.
13 Gupta P, Singh S, Goyal V, et al. Low-Dose topiramate versus lamotrigine in migraine prophylaxis (the Lotolamp study). Headache 2007;47:402-12

14 D'Andrea G, Granella F, Cadaldini M, et al. Effectiveness of lamotrigine in the prophylaxis of migraine with aura: an open pilot study. Cephalalgia 1999;19:64-6.

15 Lampl C, Buzath A, Klinger D, et al. Lamotrigine in the prophylactic treatment of migraine aura--a pilot study. Cephalalgia 1999;19:58-63.

16 Pascual J, Caminero AB, Mateos V, et al. Preventing disturbing migraine aura with lamotrigine: an open study. Headache 2004;44:1024-8.

17 Silberstein SD, Holland S, Freitag F, et al. Evidence-Based guideline update: pharmacologic treatment for episodic migraine prevention in adults: report of the Quality Standards Subcommittee of the American Academy of Neurology and the American headache Society. Neurology 2012;78:1337-45.

18 Bussone G, Diener H-C, Pfeil J, et al. Topiramate 100 mg/day in migraine prevention: a pooled analysis of double-blind randomised controlled trials. Int J Clin Pract 2005;59:961-8.

19 Worthington I, Pringsheim T, Gawel MJ, et al. Canadian headache Society guideline: acute drug therapy for migraine headache. Can $J$ Neurol Sci 2013;40:S1-3.

20 Silberstein SD, Feliu AL, Rupnow MFT, et al. Topiramate in migraine prophylaxis: long-term impact on resource utilization and cost. Headache 2007;47:500-10.

21 Hoffmann J, Charles A. Glutamate and its receptors as therapeutic targets for migraine. Neurotherapeutics 2018;15:361-70.

22 Akerman S, Goadsby PJ. Topiramate inhibits cortical spreading depression in rat and cat: impact in migraine aura. Neuroreport 2005;16:1383-7.

23 Diener H-C, Agosti R, Allais G, et al. Cessation versus continuation of 6-month migraine preventive therapy with topiramate (PROMPT) a randomised, double-blind, placebo-controlled trial. Lancet Neurol 2007:6:1054-62.

24 Reuter U, Sanchez Del Rio M, Diener H-C, et al. Migraines with and without aura and their response to preventive therapy with topiramate. Cephalalgia 2010;30:543-51.

25 Steiner TJ, Findley LJ, Yuen AW. Lamotrigine versus placebo in the prophylaxis of migraine with and without aura. Cephalalgia 1997;17:109-12.

26 Lampl C, Katsarava Z, Diener $\mathrm{H}-\mathrm{C}$, et al. Lamotrigine reduces migraine aura and migraine attacks in patients with migraine with aura. J Neurol Neurosurg Psychiatry 2005;76:1730-2.

27 Luykx J, Mason M, Ferrari MD, et al. Are migraineurs at increased risk of adverse drug responses? A meta-analytic comparison of topiramate-related adverse drug reactions in epilepsy and migraine. Clin Pharmacol Ther 2009;85:283-8.

28 Hernández-Díaz S, Smith CR, Shen A, et al. Comparative safety of antiepileptic drugs during pregnancy. Neurology 2012;78:1692-9.

29 Pariente G, Leibson T, Shulman T, et al. Pregnancy outcomes following in utero exposure to lamotrigine: a systematic review and meta-analysis. CNS Drugs 2017;31:439-50. 